\title{
Two-Step Signal Detection for MIMO-OFDM Systems without Cyclic Prefix
}

\author{
Shaodan Ma and Tung-Sang Ng, Fellow, IEEE \\ Dept. of Electrical and Electronic Engineering \\ The University of Hong Kong \\ Email: \{sdma,tsng\}@eee.hku.hk
}

\begin{abstract}
In this paper, a MIMO-OFDM system without cyclic prefix (CP) is considered and a two-step signal detection algorithm is proposed. The algorithm is based on some structural properties derived from shifting the received OFDM symbols. The first step cancels inter-carrier interference (ICI) and inter-symbol interference (ISI) with an equalizer designed using second-order statistics of the shifted received OFDM symbols. The second step detects the signals from the equalizer output in which the signals are still corrupted with multiantenna interference (MAI). In the proposed algorithm, precise knowledge of the channel length is unnecessary and only one pilot OFDM symbol is utilized to estimate the required channel state information, assuming the number of transmit antennas is smaller than the number of subcarriers in one OFDM symbol. Simulation results show that the proposed algorithm achieves comparable performance to algorithms for MIMO-OFDM system with cyclic prefix and it is robust against channel length overestimation.
\end{abstract}

\section{INTRODUCTION}

Recently, the combination of two powerful techniques, MIMO (multiple input multiple output) and OFDM (orthogonal frequency division multiplexing), has attracted a lot of research interest due to its potential for effective wireless communications [1,2]. In conventional MIMOOFDM systems operating over frequency selective fading channels, signals can be easily detected using a set of parallel one-tap linear equalizers as a cyclic prefix (CP) is generally inserted at the beginning of each OFDM symbol to reduce the frequency selective channels into flat channels on each subcarrier [1]. The length of the CP is chosen longer than the channel length to eliminate the inter-carrier interference (ICI) and inter-symbol interference (ISI). For example, in the wireless local area network (IEEE 802.11a) standard, the length of CP is $25 \%$ of an OFDM symbol duration, resulting in a significant loss in bandwidth efficiency. It is apparent that if the CP is removed, substantial gain in bandwidth efficiency can be achieved. A MIMO-OFDM system without CP (MIMO-OFDM-WCP) is therefore desirable and signal detection for MIMO-OFDMWCP systems becomes an important research topic in recent years.

As the CP is removed, ICI and ISI are introduced into the received signals. Their presence destroys the orthogonality of the subcarriers, making signal detection very difficult, if not impossible. So far there are only a few published works on this type of systems. In [3], a linear

This work was supported by the Hong Kong Research Grants Council (Grant No.: 7160/06E). smoothing signal detection algorithm is proposed based on second-order statistics (SOS) of the received signals. It requires precise estimation of the channel length, which is difficult to achieve in practice. The algorithms in [4,5] apply decision-feedback equalization (DFE) method in which successive decision of the previous OFDM symbol is utilized. These algorithms do have error propagation problem which will limit their performance. In [6], a twostep method (ISI cancellation and then ICI cancellation) based on successive decision of the previous OFDM symbol is proposed. This method is rather complex and also has the error propagation problem. Note that all the above algorithms are only applicable in single transmit antenna systems, and their extension to multiple transmit antenna systems is by no means straightforward.

In this paper, signal detection for a MIMO-OFDMWCP system is considered and a two-step algorithm is proposed. By modeling the system using the shifting method which is introduced for MIMO-OFDM systems with $C P$ (MIMO-OFDM-CP) in [9], some new special structural properties are derived. With these properties, it turns out that some second-order statistical matrices of the shifted received OFDM symbols are similar to that of single carrier MIMO systems. It follows that the blind SOS-based zero-forcing equalization method proposed for single carrier MIMO system [8] can be utilized here to cancel all the ICI and ISI in the first step. Then the signals are detected in the presence of multi-antenna interference (MAI) with the aid of only one pilot OFDM symbol, given that the number of transmit antennas is smaller than the number of subcarriers in the pilot OFDM symbol. In the proposed algorithm, the number of pilot OFDM symbols is less than that required in the conventional signal detection algorithm [1] for MIMOOFDM-CP systems, in which the minimum number of pilot OFDM symbols required is equal to the number of transmit antennas. In addition, precise channel length estimation is unnecessary and higher bandwidth efficiency is achieved. Simulation results indicate that the proposed algorithm achieves comparable performance to that of the conventional signal detection algorithm [1] for MIMO-OFDM-CP systems, and it is robust against channel length overestimation.

The rest of the paper is organized as follows. In Section II, the MIMO-OFDM-WCP system model is introduced. The two-step signal detection algorithm is presented in Section III and in Section IV, the performance of the proposed algorithm is demonstrated by simulation. Finally, Section V draws the conclusion. 
Some notations are used throughout this paper. Superscripts $T, *$, and \# stand for transpose, conjugate transpose and pseudo-inverse, respectively. $\mathbf{I}_{a}$ denotes the $a \times a$ identity matrix, and $\otimes$ is the Kronecker product of matrices.

\section{SYSTEM MODEL}

Consider a MIMO-OFDM-WCP system with $P$ transmit antennas and $M$ receive antennas. In order to achieve high transmission rate, the data is demultiplexed into $P$ parallel independent bit streams. Each bit stream is grouped into blocks, transformed into OFDM symbols by IFFT (inverse fast fourier transform) and transmitted through one transmit antenna. In general, a number of OFDM symbols are grouped into a packet and a header which includes pilot symbols for both timing and frequency synchronization are inserted. At the receiver, the header is discarded after processed for specific purpose. In the following, the header will not be considered further. Denote the $i$ th block signal from the $p$ th transmit antenna before IFFT as $\mathbf{b}_{i, p}=\left[b_{i, p}[0] \quad \cdots \quad b_{i, p}[N-1]\right]^{T}, p \in\{1,2, \cdots, P\}$, where $N$ is the number of subcarriers in one OFDM symbol. Without loss of generality, $b_{i, p}[n], n \in\{0,1, \cdots, N-1\}$, are assumed to be statistically independent and white with zero mean and unit variance. After IFFT, the transmitted OFDM symbol is generated as $\mathbf{s}_{i, p}=\mathbf{F}_{N} \mathbf{b}_{i, p}, p \in\{1,2, \cdots, P\}$, in which $\mathbf{s}_{i, p}=\left[\begin{array}{lll}s_{i, p}[0] & \cdots & s_{i, p}[N-1]\end{array}\right]^{T}$ and $\mathbf{F}_{N}$ is the $N \times N$ IFFT matrix with the $(n+1, k+1)$ th entry being $e^{j 2 \pi n k / N} / \sqrt{N}, n, k \in\{0,1, \cdots, N-1\}$. It is obvious that the FFT matrix is $\mathbf{F}_{N}^{*}$ and $\mathbf{F}_{N} \mathbf{F}_{N}^{*}=\mathbf{I}_{N}$. In conventional MIMOOFDM systems, a cyclic prefix is generally added at the beginning of each OFDM symbol to eliminate ICI and ISI. Here, no cyclic prefix is added, leading to higher bandwidth efficiency. The OFDM symbols from all transmit antennas are simultaneously transmitted into frequency selective fading channels. Denote the frequency selective fading channel between the $p$ th transmit antenna and the $m$ th receive antenna be $h_{p m}(l)$, which is generally modeled as a $L_{p m}$ th-order FIR filter. Assume the system is frequency and time synchronized with the aid of pilot symbols transmitted at the beginning of the data packet. The $i$ th received OFDM symbol at the $m$ th receive antenna is therefore written as

$$
\begin{gathered}
y_{i, m}[n]=\sum_{p=1}^{P} \sum_{l=0}^{L} h_{p m}(l) s_{i, p}[n-l]+w_{i, m}[n], \\
n=0,1, \cdots, N-1, m=1,2, \cdots, M,
\end{gathered}
$$

where $L$ represents the maximum channel length as $L=\max _{1 \leq p \leq P, 1 \leq m \leq M}\left(L_{p m}\right) ; h_{p m}(l)$ is zero-padded for $L_{p m}<l \leq L ; w_{i, m}[n]$ is independently and identically distributed (i.i.d.) white Gaussian noise at the $m$ th receive antenna and is uncorrelated with the transmitted signals; $s_{i, p}[n]=s_{i+1, p}[n-N]$ for $N \leq n$, and $s_{i, p}[n]=s_{i-1, p}[n+N]$ for $-N \leq n<0$. Here, the maximum channel length $L$ is generally assumed to be less than the number of subcarriers $N$. This assumption is consistent with the conventional MIMO-OFDM-CP systems where the maximum channel length is assumed to be shorter than the CP length. A practical example is IEEE 802.11a WLAN standard where the CP length is equal to $25 \%$ of $N$. By defining

$$
\begin{aligned}
& \mathbf{y}_{i}[n]=\left[\begin{array}{llll}
y_{i, 1}[n] & y_{i, 2}[n] & \cdots & y_{i, M}[n]
\end{array}\right]^{T}, \\
& \mathbf{h}_{p}(l)=\left[\begin{array}{llll}
h_{p 1}(l) & h_{p 2}(l) & \cdots & h_{p M}(l)
\end{array}\right]^{T}, \\
& \mathbf{w}_{i}[n]=\left[\begin{array}{llll}
w_{i, 1}[n] & w_{i, 2}[n] & \cdots & \left.w_{i, M}[n]\right]^{T},
\end{array}\right.
\end{aligned}
$$

(1) can be expressed in vector form as

$$
\begin{gathered}
\mathbf{y}_{i}[n]=\sum_{p=1}^{P} \sum_{l=0}^{L} \mathbf{h}_{p}(l) s_{i, p}[n-l]+\mathbf{w}_{i}[n], \\
n=0,1, \cdots, N-1 .
\end{gathered}
$$

At the receiver, signal detection is traditionally performed based on the $i$ th received OFDM symbol with $N$-sample signals as

$$
\mathbf{y}_{i}^{(0)}=\left[\begin{array}{llll}
\mathbf{y}_{i}[0]^{T} & \mathbf{y}_{i}[1]^{T} & \cdots & \mathbf{y}_{i}[N-1]^{T}
\end{array}\right]^{T} .
$$

Here the $i$ th received OFDM symbol shifted by $k$ samples is collected and modeled as

$$
\mathbf{y}_{i}^{(k)}=\mathbf{H} \mathbf{x}_{i}^{(k)}+\mathbf{w}_{i}^{(k)}, k=0, \pm 1, \pm 2, \cdots,
$$

where

$$
\begin{aligned}
& \mathbf{y}_{i}^{(k)}=\left[\begin{array}{llll}
\mathbf{y}_{i}[-k]^{T} & \mathbf{y}_{i}[-k+1]^{T} \quad \cdots \quad \mathbf{y}_{i}[N-1-k]^{T}
\end{array}\right]^{T}, \\
& \mathbf{x}_{i}^{(k)}=\left[\begin{array}{llll}
\left(\mathbf{x}_{i, 1}^{(k)}\right)^{T} & \left(\mathbf{x}_{i, 2}^{(k)}\right)^{T} & \cdots & \left(\mathbf{x}_{i, P}^{(k)}\right)^{T}
\end{array}\right]^{T}, \\
& \mathbf{x}_{i, p}^{(k)}=\left[\begin{array}{lllll}
s_{i, p}[-L-k] & \cdots & s_{i, p}[0] & \cdots & s_{i, p}[N-1-k]
\end{array}\right]^{T}, \\
& p \in\{1,2, \cdots, P\}, \\
& \mathbf{H}=\left[\begin{array}{llll}
\mathbf{H}_{1} & \mathbf{H}_{2} & \cdots & \mathbf{H}_{P}
\end{array}\right], \\
& \mathbf{H}_{p}=\left[\begin{array}{ccccccc}
\mathbf{h}_{p}(L) & \mathbf{h}_{p}(L-1) & \cdots & \mathbf{h}_{p}(0) & \mathbf{0} & \cdots & \mathbf{0} \\
\mathbf{0} & \mathbf{h}_{p}(L) & \mathbf{h}_{p}(L-1) & \cdots & \mathbf{h}_{p}(0) & \ddots & \mathbf{0} \\
\vdots & \ddots & \ddots & \ddots & \ddots & \ddots & \vdots \\
\mathbf{0} & \cdots & \mathbf{0} & \mathbf{h}_{p}(L) & \mathbf{h}_{p}(L-1) & \cdots & \mathbf{h}_{p}(0)
\end{array}\right] \\
& p \in\{1,2, \cdots, P\} \text {, } \\
& \left.\mathbf{w}_{i}^{(k)}=\left[\begin{array}{llll}
\mathbf{w}_{i}[-k]^{T} & \mathbf{w}_{i}[-k+1]^{T} & \cdots & \mathbf{w}_{i}[N-1-k
\end{array}\right]^{T}\right]^{T} .
\end{aligned}
$$

In (8), when $-N \leq n<0, \mathbf{y}_{i}[n]$ represents the signal in the $(i-1)$ th received OFDM symbol and is equal to $\mathbf{y}_{i-1}[N+n]$. Similarly, when $N \leq n, \mathbf{y}_{i}[n]$ represents the signal in the $(i+1)$ th received OFDM symbol and is equal to $\mathbf{y}_{i+1}[n-N]$. The $M N \times(N+L) P$ matrix $\mathbf{H}$ in (11) is the so-called channel convolution matrix, and is generally assumed to have full column rank after removing all-zero columns. This is a reasonable assumption as the number of receive antennas, $M$, can be chosen to satisfy $M \geq(L+N) P / N$, such that the matrix $\mathbf{H}$ has more rows than columns. Therefore, in practical channels, it is most likely to be of full 
column rank (otherwise this can still be achieved by simple artificial loading of the matrix) and has the property [7]

$$
\mathbf{H}^{*}\left(\mathbf{H H}^{*}\right)^{\#} \mathbf{H}=\mathbf{A}_{(N+L) P},
$$

in which $\mathbf{A}_{(N+L) P}$ is an $(N+L) P \times(N+L) P$ identity matrix with zero rows corresponding to the all-zero columns of $\mathbf{H}$.

In the shifted received signal model (7), the transmitted signal vector $\mathbf{x}_{i, p}^{(k)}(10)$ contains the $(i-1)$ th OFDM symbol signals $\left(\left[s_{i, p}[-L-k] \cdots s_{i, p}[-1]\right]\right)$ and the $i$ th OFDM symbol signals $\left(\left[\begin{array}{lll}s_{i, p}[0] & \cdots & s_{i, p}[N-1-k]\end{array}\right]\right)$ when $0 \leq k \leq N-L$. It can be rewritten in terms of the signals before IFFT $\mathbf{b}_{i, p}$ as

$$
\mathbf{x}_{i, p}^{(k)}=\mathbf{F}_{2 N}^{(k)} \mathbf{c}_{i, p}, 0 \leq k \leq N-L, p \in\{1,2, \cdots, P\},
$$

where

$$
\mathbf{c}_{i, p}=\left[\begin{array}{ll}
\mathbf{b}_{(i-1), p}^{T} & \mathbf{b}_{i, p}^{T}
\end{array}\right]^{T}
$$

and

$$
\mathbf{F}_{2 N}^{(k)}=\left[\begin{array}{cc}
\mathbf{F}_{N}(N-L-k+1: N) & \mathbf{0} \\
\mathbf{0} & \mathbf{F}_{N}(1: N-k)
\end{array}\right] .
$$

In (17), $\mathbf{F}_{N}(a: b)$ denotes a submatrix composed of the $a$ th to the $b$ th row of $\mathbf{F}_{N}$. Since each column (row) of the IFFT matrix $\mathbf{F}_{N}$ is orthogonal to all other columns (rows), it is apparent that the matrix $\mathbf{F}_{2 N}^{(k)}$ in (17) satisfies

$$
\begin{gathered}
\mathbf{F}_{2 N}^{(0)} \mathbf{F}_{2 N}^{(0)^{*}}=\mathbf{J}^{0}=\mathbf{I}_{N+L}, \\
\mathbf{F}_{2 N}^{(k)} \mathbf{F}_{2 N}^{(0)^{*}}=\mathbf{J}^{k}, 0 \leq k \leq N-L, \\
\mathbf{F}_{2 N}^{(0)} \mathbf{F}_{2 N}^{(k) *}=\mathbf{J}^{-k}, 0 \leq k \leq N-L,
\end{gathered}
$$

where $\mathbf{J}^{k}$ denotes a $(N+L) \times(N+L)$ matrix with zero entries except along the lower $k$ th subdiagonal, in which the entries are one, and $\mathbf{J}^{-k}$ is equal to $\left(\mathbf{J}^{k}\right)^{*}$. Here the structural properties of (15) and (18)-(20) are essential for the signal detection algorithm to be derived in the following section.

\section{Two-STEP SignAL DETECTION}

From the shifted received signal model (7), it is obvious that $\mathbf{y}_{i}^{(k)}$ includes $(N+L)$ path signals from each transmit antenna. ISI, ICI and MAI simultaneously exist in $\mathbf{y}_{i}^{(k)}$. In order to detect the transmitted signals from $\mathbf{y}_{i}^{(k)}$, a two-step signal detection algorithm is proposed here.

To simplify the algorithm derivation, zero noise is first assumed. The effect of noise on the algorithm is then examined. In the absence of noise, $\mathbf{y}_{i}^{(k)}$ can be expressed as

$$
\mathbf{y}_{i}^{(k)}=\mathbf{H x}_{i}^{(k)}, k=0, \pm 1, \pm 2, \cdots .
$$

\section{A. Step 1: Blind ICI and ISI cancellation}

Now, consider some second-order statistical matrices of the shifted $i$ th received OFDM symbol $\mathbf{y}_{i}^{(k)}$,

$$
\mathbf{R}_{y}(K)=E\left\{\mathbf{y}_{i}^{(K)} \mathbf{y}_{i}^{(0) *}\right\}=E\left\{\mathbf{H} \mathbf{x}_{i}^{(K)} \mathbf{x}_{i}^{(0) *} \mathbf{H}^{*}\right\},
$$

$$
\mathbf{R}_{y}(-K)=E\left\{\mathbf{y}_{i}^{(0)} \mathbf{y}_{i}^{(K)^{*}}\right\}=E\left\{\mathbf{H} \mathbf{x}_{i}^{(0)} \mathbf{x}_{i}^{(K)^{*}} \mathbf{H}^{*}\right\},
$$

and assume $0 \leq K \leq N-L$. It follows from the signals defined in (9) and the property (15) that

$$
\begin{aligned}
& \mathbf{R}_{y}(K)=E\left\{\mathbf{H} \mathbf{x}_{i}^{(K)} \mathbf{x}_{i}^{(0)^{*}} \mathbf{H}^{*}\right\} \\
& =\mathbf{H}\left[\begin{array}{ccc}
\mathbf{F}_{2 N}^{(K)} E\left\{\mathbf{c}_{i, 1} \mathbf{c}_{i, 1}^{*}\right\} \mathbf{F}_{2 N}^{(0)^{*}} & \mathbf{0} & \mathbf{0} \\
\mathbf{0} & \ddots & \mathbf{0} \\
\mathbf{0} & \mathbf{0} & \mathbf{F}_{2 N}^{(K)} E\left\{\mathbf{c}_{i, P} \mathbf{c}_{i, P}^{*}\right\} \mathbf{F}_{2 N}^{(0) *}
\end{array}\right] \mathbf{H}^{*}, \\
& \mathbf{R}_{y}(-K)=E\left\{\mathbf{H} \mathbf{x}_{i}^{(0)} \mathbf{x}_{i}^{\left.(K)^{*} \mathbf{H}^{*}\right\}}\right. \\
& =\mathbf{H}\left[\begin{array}{ccc}
\mathbf{F}_{2 N}^{(0)} E\left\{\mathbf{c}_{i, 1} \mathbf{c}_{i, 1}^{*}\right\} \mathbf{F}_{2 N}^{(K)^{*}} & \mathbf{0} & \mathbf{0} \\
\mathbf{0} & \ddots & \mathbf{0} \\
\mathbf{0} & \mathbf{0} & \mathbf{F}_{2 N}^{(0)} E\left\{\mathbf{c}_{i, P} \mathbf{c}_{i, P}^{*}\right\} \mathbf{F}_{2 N}^{(K) *}
\end{array}\right] \mathbf{H}^{*} .
\end{aligned}
$$

As the signals before IFFT are assumed to be statistically independent and white with zero mean and unit variance, $E\left\{\mathbf{c}_{i, p} \mathbf{c}_{i, p}^{*}\right\}=\mathbf{I}_{2 N}, p \in\{1,2, \cdots, P\}$. It follows that

$$
\begin{gathered}
\mathbf{R}_{y}(K)=E\left\{\mathbf{H} \mathbf{x}_{i}^{(K)} \mathbf{x}_{i}^{(0)^{*}} \mathbf{H}^{*}\right\}=\mathbf{H}\left(\mathbf{I}_{P} \otimes \mathbf{F}_{2 N}^{(K)} \mathbf{F}_{2 N}^{(0)^{*}}\right) \mathbf{H}^{*}, \\
\mathbf{R}_{y}(-K)=E\left\{\mathbf{H} \mathbf{x}_{i}^{(0)} \mathbf{x}_{i}^{(K)^{*}} \mathbf{H}^{*}\right\}=\mathbf{H}\left(\mathbf{I}_{P} \otimes \mathbf{F}_{2 N}^{(0)} \mathbf{F}_{2 N}^{(K)}\right) \mathbf{H}^{*} .
\end{gathered}
$$

Substituting (18)-(20) into (26) and (27),

$$
\begin{gathered}
\mathbf{R}_{y}(K)=E\left\{\mathbf{H} \mathbf{x}_{i}^{(K)} \mathbf{x}_{i}^{(0)^{*}} \mathbf{H}^{*}\right\}=\mathbf{H}\left(\mathbf{I}_{P} \otimes \mathbf{J}^{K}\right) \mathbf{H}^{*}, \\
\mathbf{R}_{y}(-K)=E\left\{\mathbf{H} \mathbf{x}_{i}^{(0)} \mathbf{x}_{i}^{(K)^{*}} \mathbf{H}^{*}\right\}=\mathbf{H}\left(\mathbf{I}_{P} \otimes \mathbf{J}^{-K}\right) \mathbf{H}^{*}, \\
\mathbf{R}_{y}(0)=E\left\{\mathbf{H} \mathbf{x}_{i}^{(0)} \mathbf{x}_{i}^{(0) *} \mathbf{H}^{*}\right\}=\mathbf{H}\left(\mathbf{I}_{P} \otimes \mathbf{I}_{N+L}\right) \mathbf{H}^{*}=\mathbf{H} \mathbf{H}^{*} .
\end{gathered}
$$

It is found that the second-order statistical matrices (28)-(30) are similar to that of single carrier MIMO system [8]. As a result, the SOS-based zero-forcing equalization method for single-carrier MIMO system [8] can be readily applied here. An equalizer is thus constructed as

$$
\mathbf{G}_{K}=\mathbf{U}_{K}-\mathbf{U}_{K+1},
$$

where

$$
\mathbf{U}_{K}=\mathbf{R}_{y}(-K) \mathbf{R}_{y}(0)^{\#} \mathbf{R}_{y}(K) \mathbf{R}_{y}(0)^{\#} .
$$

Using the property of the channel matrix $\mathbf{H}$ in (14) and applying (28)-(30), the matrix $\mathbf{U}_{K}$ becomes

$$
\mathbf{U}_{K}=\mathbf{H}\left(\mathbf{I}_{P} \otimes \mathbf{J}^{-K} \mathbf{J}^{K}\right) \mathbf{H}^{*}\left(\mathbf{H H}^{*}\right)^{\#} .
$$

Substituting (33) into (31), the equalizer is equivalent to

$$
\mathbf{G}_{K}=\mathbf{H}\left(\mathbf{I}_{P} \otimes\left(\mathbf{J}^{-K} \mathbf{J}^{K}-\mathbf{J}^{-(K+1)} \mathbf{J}^{(K+1)}\right)\right) \mathbf{H}^{*}\left(\mathbf{H} \mathbf{H}^{*}\right)^{\#} \text {. }
$$

It is observed that the matrix $\mathbf{J}^{K}$ satisfies [8]

$$
\begin{gathered}
\mathbf{J}^{-K} \mathbf{J}^{K}=\left[\begin{array}{cc}
\mathbf{I}_{(N+L-K)} & \mathbf{0}_{(N+L-K) \times K} \\
\mathbf{0}_{K \times(N+L-K)} & \mathbf{0}_{K \times K}
\end{array}\right], \\
\mathbf{J}^{-K} \mathbf{J}^{K}-\mathbf{J}^{-(K+1)} \mathbf{J}^{(K+1)} \\
=\left[\begin{array}{ccc}
\mathbf{0}_{(N+L-K-1) \times(N+L-K-1)} & \mathbf{0}_{(N+L-K-1) \times 1} & \mathbf{0}_{(N+L-K-1) \times K} \\
\mathbf{0}_{1 \times(N+L-K-1)} & 1 & \mathbf{0}_{1 \times K} \\
\mathbf{0}_{K \times(N+L-K-1)} & \mathbf{0}_{K \times 1} & \mathbf{0}_{K \times K}
\end{array}\right],
\end{gathered}
$$

where $\mathbf{0}_{a \times b}$ is an $a \times b$ zero matrix. The matrix $\mathbf{J}^{-K} \mathbf{J}^{K}-\mathbf{J}^{-(K+1)} \mathbf{J}^{(K+1)}$ in (36) is zero except the 
$(N+L-K, N+L-K)$ entry which is one. From this observation, it follows that

$$
\begin{aligned}
& \mathbf{G}_{K}=\left[\begin{array}{lll}
\mathbf{0}_{M N \times(N+L-K-1)} & \mathbf{H}_{1}(N+L-K) & \mathbf{0}_{M N \times K}
\end{array}\right. \\
& \left.\begin{array}{llll}
\cdots & \mathbf{0}_{M N \times(N+L-K-1)} & \mathbf{H}_{P}(N+L-K) & \mathbf{0}_{M N \times K}
\end{array}\right] \mathbf{H}^{*}\left(\mathbf{H H}^{*}\right)^{\#} .
\end{aligned}
$$

In $(37), \mathbf{H}_{p}(a)$ denotes the $a$ th column of the matrix $\mathbf{H}_{p}$, $p=1,2, \cdots, P$.

Applying the equalizer $\mathbf{G}_{K}$ into the shifted $i$ th received OFDM symbol $\mathbf{y}_{i}^{(k)}(21)$, we have

$$
\begin{aligned}
& \mathbf{o}_{i, K}^{(k)}=\mathbf{G}_{K} \mathbf{y}_{i}^{(k)}=\left(\mathbf{U}_{K}-\mathbf{U}_{K+1}\right) \mathbf{H} \mathbf{x}_{i}^{(k)} \\
& =\left[\begin{array}{lll}
\mathbf{0}_{M N \times(N+L-K-1)} & \mathbf{H}_{1}(N+L-K) & \mathbf{0}_{M N \times K}
\end{array}\right. \\
& \left.\begin{array}{llll}
\cdots & \mathbf{0}_{M N \times(N+L-K-1)} & \mathbf{H}_{P}(N+L-K) & \mathbf{0}_{M N \times K}
\end{array}\right] \mathbf{H}^{*}\left(\mathbf{H H}^{*}\right)^{\#} \mathbf{H} \mathbf{x}_{i}^{(k)} \\
& k=0, \pm 1, \pm 2, \cdots \text {. }
\end{aligned}
$$

Using (9), (10) and (14), the equalizer output $\mathbf{o}_{i, K}^{(k)}$ becomes

$$
\mathbf{o}_{i, K}^{(k)}=\overline{\mathbf{H}}_{\text {part }}^{(K)} \overline{\mathbf{x}}_{i, \text { part }}^{(k)}, k=0, \pm 1, \pm 2, \cdots,
$$

where

$$
\begin{aligned}
& \overline{\mathbf{H}}_{\text {part }}^{(K)}=\left[\mathbf{H}_{1}(N+L-K) \quad \mathbf{H}_{2}(N+L-K)\right. \\
& \left.\cdots \quad \mathbf{H}_{P}(N+L-K)\right] \\
& \overline{\mathbf{x}}_{i, \text { part }}^{(k)}=\left[s_{i, 1}[N-1-k-K] \quad s_{i, 2}[N-1-k-K]\right. \\
& \left.\cdots \quad s_{i, P}[N-1-k-K]\right]^{T}
\end{aligned}
$$

It is apparent that only one path signal from each transmit antenna is retained in the equalizer output. It means that both ICI and ISI are cancelled by the equalizer.

\section{B. Step 2: Signal detection in the presence of MAI}

Although the equalizer has cancelled ICI and ISI, MAI still exists in the equalizer output $\mathbf{o}_{i, K}^{(k)}$. To proceed with signal detection, we notice that knowledge of the matrix $\overline{\mathbf{H}}_{\text {part }}^{(K)}$ in (40) is required. For better performance, pilots will be utilized to estimate $\overline{\mathbf{H}}_{\text {part }}^{(K)}$.

As $\overline{\mathbf{H}}_{\text {part }}^{(K)}$ is an $M N \times P$ matrix, its estimation requires at least $P$ sets of equation (39). Choosing the $P$ sets of equation (39) and writing them in matrix form as

$$
\mathbf{O}_{\text {pilot }}=\overline{\mathbf{H}}_{\text {part }}^{(K)} \mathbf{X}_{\text {pilot }},
$$

where

$$
\begin{gathered}
\mathbf{O}_{\text {pilot }}=\left[\begin{array}{llll}
\mathbf{o}_{i, K}^{(0)} & \mathbf{o}_{i, K}^{(1)} & \cdots & \mathbf{o}_{i, K}^{(P)}
\end{array}\right] \\
\mathbf{X}_{\text {pilot }}=\left[\begin{array}{llll}
\overline{\mathbf{x}}_{i, \text { part }}^{(0)} & \overline{\mathbf{x}}_{i, \text { part }}^{(1)} & \cdots & \overline{\mathbf{x}}_{i, \text { part }}^{(P)}
\end{array}\right],
\end{gathered}
$$

an estimation of $\overline{\mathbf{H}}_{\text {part }}^{(K)}$ can be easily obtained based on leastsquares criteria as

$$
\hat{\overline{\mathbf{H}}}_{\text {part }}^{(K)}=\mathbf{O}_{\text {pilot }} \mathbf{X}_{\text {pilot }}^{*}\left(\mathbf{X}_{\text {pilot }} \mathbf{X}_{\text {pilot }}^{*}\right)^{\#} .
$$

In (43), the $P \times P$ matrix $\mathbf{X}_{\text {pilot }}$ is the so-called pilot matrix and includes $P$ samples from each transmit antenna. In order to obtain a unique estimation of $\overline{\mathbf{H}}_{\text {part }}^{(K)}$, it must be selected to have full rank.
With knowledge of the matrix $\overline{\mathbf{H}}_{\text {part }}^{(K)}$, a number of algorithms [1] can be applied to detect the transmitted signal $\overline{\mathbf{x}}_{i, \text { part }}^{(k)}$ as the equalizer output (39) only contains MAI. Here, the least-squares detection method is selected for its simplicity and the transmitted signal is detected as

$$
\hat{\overline{\mathbf{x}}}_{i, \text { part }}^{(k)}=\mathbf{G}_{L S} \mathbf{o}_{i, K}^{(k)}, k=0, \pm 1, \pm 2, \cdots .
$$

where $\mathbf{G}_{L S}$ is an one-tap linear equalizer given by $\mathbf{G}_{L S}=\left(\overline{\mathbf{H}}_{\text {part }}^{(K) *} \overline{\mathbf{H}}_{\text {part }}^{(K)}\right)^{-1} \overline{\mathbf{H}}_{\text {part }}^{(K) *} \quad$. $\quad$ It is obvious that $s_{i, p}[N-1-k-K], p \in\{1,2, \cdots, P\}$, is the signal after IFFT (see (41)). The signals $b_{i, p}[n]$ can be recovered by performing FFT to the signals $s_{i, p}[n], n \in\{0,1, \cdots, N-1\}$, which can be obtained from (45) by setting the parameter $k=N-K-1, N-K-2, \cdots,-K$.

Apparently, only $P$ pilot samples from each transmit antenna are utilized in the pilot matrix $\mathbf{X}_{\text {pilot }}$ (43) for signal detection. When $P$ is less than the number of subcarriers $N$ in one OFDM symbol (which is usually the real situation), one pilot OFDM symbol is sufficient. The number of pilot OFDM symbols required here is less than that required in the conventional signal detection algorithm [1] for MIMO-OFDM-CP system, which needs at least $P$ pilot OFDM symbols and requires CP to eliminate ICI and ISI. Here the pilot OFDM symbol for channel-state estimation can be inserted as part of the header in a packet for slow fading channels or can be inserted at regular intervals within a packet for fast fading channels.

\section{Effect of channel noise}

Up to now, the algorithm is derived under the zero-noise assumption. When the additive white channel noise with variance $\sigma^{2}$ is presented, the second-order statistical matrices of the shifted $i$ th received OFDM symbol becomes

$$
\begin{aligned}
\mathbf{R}_{y}(K)= & E\left\{\mathbf{y}_{i}^{(K)} \mathbf{y}_{i}^{(0)^{*}}\right\} \\
= & E\left\{\mathbf{H} \mathbf{x}_{i}^{(K)} \mathbf{x}_{i}^{(0)^{*}} \mathbf{H}^{*}\right\}+\sigma^{2}\left(\mathbf{I}_{M} \otimes \overline{\mathbf{J}}^{K}\right), \\
\mathbf{R}_{y}(-K)= & E\left\{\mathbf{y}_{i}^{(0)} \mathbf{y}_{i}^{(K)^{*}}\right\} \\
= & E\left\{\mathbf{H} \mathbf{x}_{i}^{(0)} \mathbf{x}_{i}^{(K)^{*}} \mathbf{H}^{*}\right\}+\sigma^{2}\left(\mathbf{I}_{M} \otimes \overline{\mathbf{J}}^{-K}\right), \\
& \mathbf{R}_{y}(0)=E\left\{\mathbf{y}_{i}^{(0)} \mathbf{y}_{i}^{(0) *}\right\} \\
= & \mathbf{H} \mathbf{H}^{*}+\sigma^{2} \mathbf{I}_{M N}
\end{aligned}
$$

where $\overline{\mathbf{J}}$ is a $N \times N$ matrix with the same structure as the matrix $\mathbf{J}$. From (48), it is well-known that $\sigma^{2}$ is the least eigenvalue of the matrix $\mathbf{R}_{y}(0)$ [2]. After estimating the noise variance $\sigma^{2}$, its effect can be subtracted from the second-order statistical matrices in (46) - (48). In general, error will exist in the estimation of $\sigma^{2}$ and therefore it is not recommended to directly subtract the noise contribution (which often results in poorer performance). In the simulation in Section IV, the noise contribution is not subtracted from the second-order statistical matrices in the 
first step (blind ICI and ISI cancellation). For the second step (signal detection in the presence of MAI), pilots are utilized to estimate the matrix $\overline{\mathbf{H}}_{\text {part }}^{(K)}$ and MMSE (minimum mean square error) criteria can be exploited to take the noise into account, instead of Least-Squares criteria. Simulation results in the next section will show the effect of noise on the system performance.

\section{Implementation}

In practice, the second-order statistical matrices $\left(\mathbf{R}_{y}(0), \mathbf{R}_{y}(K)\right.$ and $\left.\mathbf{R}_{y}(-K)\right)$ can only be computed from some finite number of the received OFDM symbols. We use the most commonly used method to approximate them as

$$
\begin{gathered}
\mathbf{R}_{y}(K) \approx \frac{1}{N_{s}} \sum_{i=1}^{N_{s}} \mathbf{y}_{i}^{(K)} \mathbf{y}_{i}^{(0)^{*}}, \mathbf{R}_{y}(-K) \approx \frac{1}{N_{s}} \sum_{i=1}^{N_{s}} \mathbf{y}_{i}^{(0)} \mathbf{y}_{i}^{(K)^{*}}, \\
\mathbf{R}_{y}(0) \approx \frac{1}{N_{s}} \sum_{i=1}^{N_{s}} \mathbf{y}_{i}^{(0)} \mathbf{y}_{i}^{(0)^{*}}
\end{gathered}
$$

where $N_{s}$ is the number of OFDM symbols used. We also assume that an upper bound of the maximum channel length, that is, a number $L_{u p p}$ such that $L_{u p p} \geq L$, is known or estimated. The implementation of the proposed algorithm is summarized as follows.

Algorithm: Two-step signal detection for MIMO-OFDM systems without cyclic prefix

Step 1): Choose the parameter $K$ such that $0 \leq K \leq N-L_{u p p}$. Compute the second-order statistical matrices $\left(\mathbf{R}_{y}(0), \mathbf{R}_{y}(K)\right.$ and $\left.\mathbf{R}_{y}(-K)\right)$.

Step 2): Form the equalization matrix $\mathbf{G}_{K}$ defined in (31).

Step 3): Perform equalization as $\mathbf{o}_{i, K}^{(k)}=\mathbf{G}_{K} \mathbf{y}_{i}^{(k)}$, $k=N-K-1, N-K-2, \cdots,-K$.

Step 4): Collect the equalizer outputs as $\mathbf{O}_{\text {pilot }}$. Estimate the matrix $\overline{\mathbf{H}}_{\text {part }}^{(K)}$ using (44).

Step 5): Detect the signals from the equalizer outputs as $\hat{\overline{\mathbf{x}}}_{i, \text { part }}^{(k)}=\left(\overline{\mathbf{H}}_{\text {part }}^{(K)}{ }^{*} \overline{\mathbf{H}}_{\text {part }}^{(K)}\right)^{-1} \overline{\mathbf{H}}_{\text {part }}^{(K)^{*}} \mathbf{o}_{i, K}^{(k)}$ $k=N-K-1, N-K-2, \cdots,-K$.

Step 6): Recover $b_{i, p}[n], p=1,2, \cdots, P$, by performing FFT to the signals $s_{i, p}[n], n \in\{0,1, \cdots, N-1\}$, which is obtained from $\hat{\overline{\mathbf{x}}}_{i, \text { part }}^{(k)}$.

\section{SIMULATION RESULTS}

The performance of the proposed signal detection algorithm has been investigated through computer simulations. In the following examples, a MIMO-OFDMWCP system with $P=2$ transmit antennas and $M=3$ receive antennas $(2 \times 3$ system $)$ was considered. The number of subcarriers in one OFDM symbol was set as $N=64$. The performance measure, BER (bit error rate), was computed by averaging the results over 100 Monte Carlo realizations. In each run: (1) a data packet with 200 random OFDM symbols was transmitted from each antenna through the wireless channel with random Gaussian noise; (2) all transmitted signals were modulated by QPSK scheme; (3) the frequency selective fading channel responses were randomly generated with Rayleigh distribution function. Signal-noise-ratio ( $S N R$ ) was defined as

$$
S N R=\frac{\sum_{m=1}^{M} E\left\{\left|\sum_{p=1}^{P} \sum_{l=0}^{L} h_{p m}(l) s_{i, p}[n-l]\right|^{2}\right\}}{\sum_{m=1}^{M} E\left\{\left|w_{i, m}[n]\right|^{2}\right\}}
$$

and the second-order statistical matrices were computed from 200 OFDM symbols.

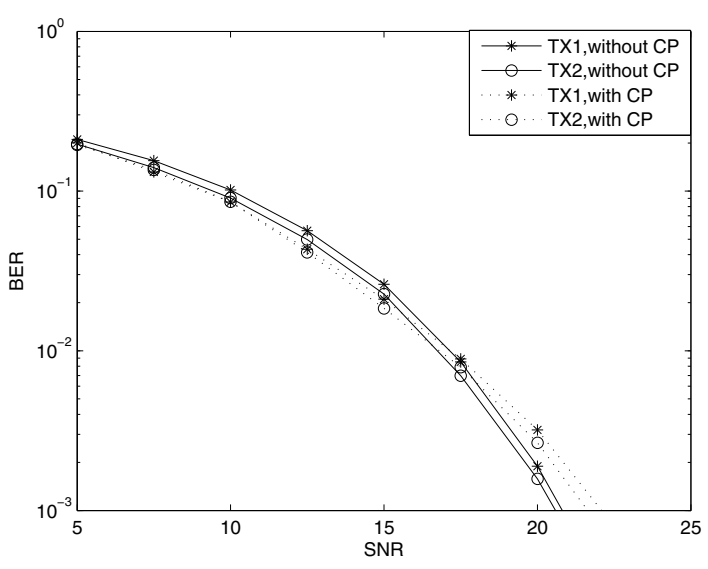

Fig. 1 BER vs SNR for $L=6$

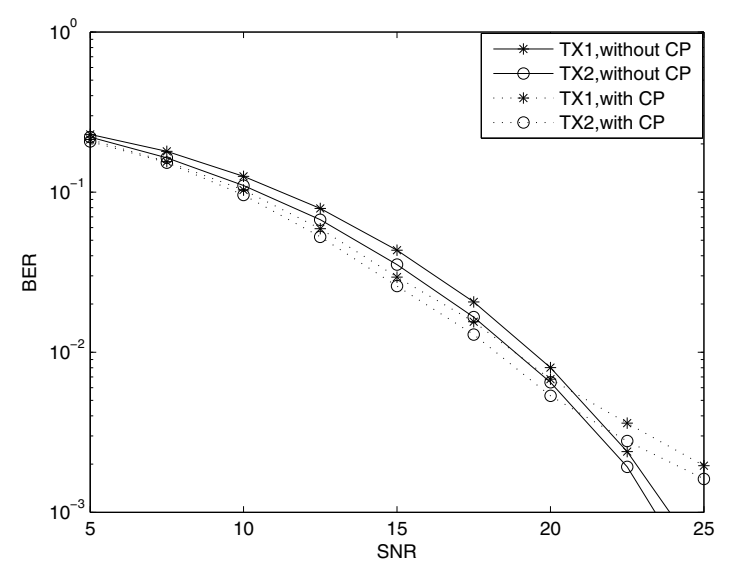

Fig. 2 BER vs SNR for $L=8$

\section{A. Effect of SNR}

For comparison, the conventional signal detection algorithm [1] was also implemented for a MIMO-OFDM-CP system. In this system, a CP with length of $25 \%$ of one OFDM symbol, that is $25 \% \times N=16$, was inserted at the beginning of each OFDM symbol to eliminate ICI and ISI. The signals were detected by a set of parallel one-tap leastsquares linear equalizers on each subcarrier after FFT. The number of pilot OFDM symbols utilized was equal to $P=2$, while only one pilot OFDM symbol was utilized in the proposed algorithm. The parameter $K$ in the proposed 
algorithm was chosen as $N / 2$.

Two cases ( $L=6$ and $L=8$ ) were considered. Results are shown in Fig. 1 and Fig. 2, respectively. It is obvious that the proposed algorithm performs slightly worse than the conventional algorithm [1] for MIMO-OFDM-CP systems when $S N R$ is low, while its performance is slightly better than that of the conventional algorithm [1] for MIMOOFDM-CP systems when $S N R$ is high. It demonstrates that the proposed algorithm for MIMO-OFDM-WCP systems can achieve comparable performance to that of the conventional signal detection algorithm [1] for MIMOOFDM-CP systems, but with higher bandwidth efficiency as $\mathrm{CP}$ is removed.

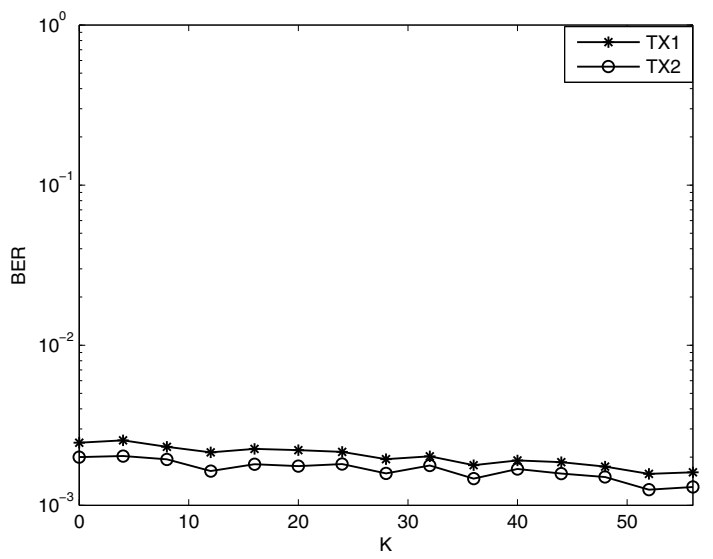

Fig. 3 Effect of $K$ for $L=6$

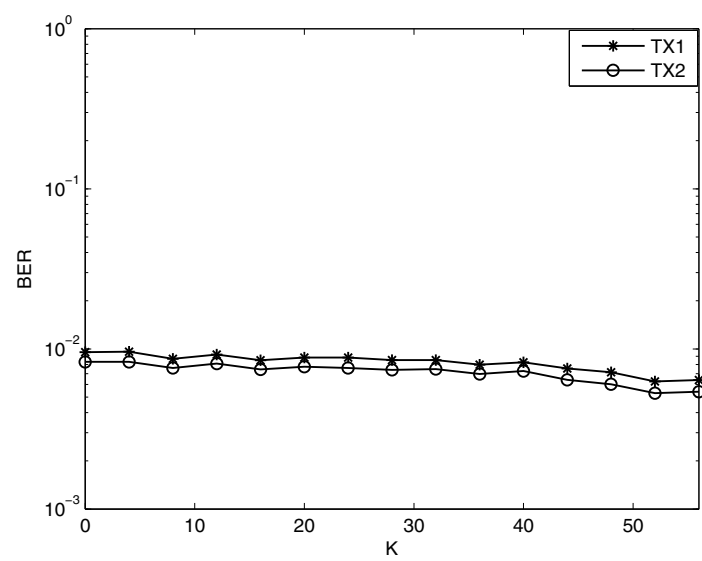

Fig. 4 Effect of $K$ for $L=8$

\section{B. Effect of the parameter $K$}

In the proposed algorithm, the parameter $K$ must be chosen such that $0 \leq K \leq N-L$. Here, it was varied from 0 to $N-L$ to test its effect on the proposed algorithm. Results are shown in Fig. 3 and Fig. 4 for the cases where $L=6$ and $L=8$, respectively. In these simulations, $S N R=20 \mathrm{~dB}$. There is little variation in their performance when $K$ varies and it indicates that the proposed algorithm is not sensitive to the parameter $K$.

\section{Effect of channel length overestimation}

The maximum channel length $L$ in the proposed algorithm determines the range of the parameter $K$, that is $K \in[0, N-L]$. The error in the estimation of $L$ only affects the system performance via the value of $K$. When $L$ is overestimated, smaller $K$ may be chosen as $K$ must lie in the range $[0, N-L]$. From the results in Fig. 3 and 4, it is clear that the performance of the proposed algorithm is not affected by the value of $K$, which in turn implies that the proposed algorithm is robust against channel length overestimation.

\section{CONCLUSION}

In this paper, a two-step signal detection algorithm for MIMO-OFDM-WCP systems has been proposed. The algorithm takes advantage of some structural properties of the shifted received OFDM symbols. An equalizer has been designed in the first step to cancel the ICI and ISI based on SOS of the received signals. Signal detection has been achieved in the second step from the equalizer output with the aid of one pilot OFDM symbol. Exact knowledge of the maximum channel length is unnecessary and higher bandwidth efficiency is achieved as CP is removed. Simulations have shown that the proposed algorithm achieves comparable performance to that of MIMO-OFDM$\mathrm{CP}$ systems and is also robust against channel length overestimation.

\section{REFERENCE}

[1] A. Van Zelst, and T. C. W. Schenk, "Implementation of a MIMO OFDM-based wireless LAN system", IEEE Trans. on Signal Processing, vol. 52, pp. 483-494, Feb. 2004

[2] Yonghong Zeng and T.S. Ng, "A Semi-Blind Channel Estimation Method For Multiuser Multiantenna OFDM Systems", IEEE Transactions on Signal Processing, vol. 52, pp. 1419-1429, May 2004

[3] X. D. Yue, and H. H. Fan, "Linear smoothing method for blind equalization of OFDM systems without cyclic prefix", Proceeding of WCNC 2004, pp. 2313-2316, Mar. 2004

[4] C. Wang, and Z. Zhou, "A new detection algorithm for OFDM system without cyclic prefix", Proceeding of CAS Symp. 2004, pp. 453-456, May 2004

[5] X. Huang, and $\mathrm{H}$. Bi, "Blind channel identification and equalization in OFDM system without cyclic prefix", Proceeding of ICCT 2003, pp. 1919-1921, Apr. 2003

[6] M. Toeltsch, and A. F. Molisch, "Efficient OFDM transmission without cyclic prefix over frequency-selective channels", Proceeding of PIMRC 2000, pp. 1363-1367, Sep. 2000

[7] G. H. Golub and C. F. Van Loan, Matrix Computations, $3^{\text {rd }}$ ed. Baltimore, MD: Johns Hopkins Univ. Press, 1996

[8] J. Zhu, Z. Ding and X.-R. Cao, "Column-anchored zeroforcing blind equalization for multiuser wireless FIR channels," IEEE J. Select. Areas Commun., vol. 17, pp. 411-423, Mar. 1999

[9] S. D. Ma and T.S. Ng, "Time-domain signal detection based on second-order statistics for MIMO-OFDM systems", IEEE Trans. on Signal Processing, vol. 55, pp. 1150-1158, Mar. 2007 